\title{
Reabilitação estética e funcional com resinas compostas prensadas
}

\author{
Aesthetic and functional rehabilitation with pressed composite resins \\ Rehabilitación estética y funcional con resinas compuestas prensadas
}

\author{
Anderson Seitiro Miyazato \\ ORCID: https://orcid.org/0000-0002-4821-3748 \\ Universidade Pitágoras UNOPAR, Brasil \\ E-mail: anderson_miyazato@hotmail.com \\ Renato Azeredo da Silva \\ ORCID: https://orcid.org/0000-0003-2466-4509 \\ Cirurgião Dentista, Brasil \\ E-mail: renato@clarus.odo.br \\ Sandrine Bittencourt Berger \\ ORCID: https://orcid.org/0000-0002-7915-3207 \\ Universidade Pitágoras UNOPAR, Brasil \\ E-mail: berger.sandrine@gmail.com \\ Danielle Gregorio \\ ORCID: https://orcid.org/0000-0002-0098-624X \\ Universidade Pitágoras UNOPAR, Brasil \\ E-mail: daanigregorio@ hotmail.com \\ Lucia Gloria Diana Aguilar Pizzurno \\ ORCID: https://orcid.org/0000-0002-9737-4249 \\ Universidade Pitágoras UNOPAR, Brasil \\ E-mail: dianita.982@hotmail.com \\ Alejandra Hortencia Miranda González \\ ORCID: https://orcid.org/0000-0003-2982-8736 \\ Universidade Pitágoras UNOPAR, Brasil \\ E-mail: alejandra.horten@ anhanguera.com \\ Murilo Baena Lopes \\ ORCID: https://orcid.org/0000-0001-6198-7695 \\ Universidade Pitágoras UNOPAR, Brasil \\ E-mail: baenalopes@gmail.com \\ Ricardo Danil Guiraldo \\ ORCID: https://orcid.org/0000-0002-1625-3120 \\ Universidade Pitágoras UNOPAR, Brasil \\ E-mail: rdguiraldo@gmail.com
}

\begin{abstract}
Resumo
O atual desenvolvimento e confiabilidade dos sistemas adesivos e das resinas compostas oferecem boas opções para tratamento e manejo das perdas de estruturas dentárias. Pacientes com desgastes mínimo, moderado ou severo podem ser tratados de forma minimamente invasiva com restaurações adesivas em resina composta. Assim, de acordo com os conhecimentos a partir da prática clínica e com base na literatura científica sobre o tema abordado, e considerando as diferentes possibilidades de condução do caso apresentado, propôs-se a realização da reabilitação da maxila e de mandíbula com o uso da Reabilitação Estética e Funcional com Resinas Compostas Prensadas (REFRCP) com resina composta de viscosidade convencional aquecida e resina composta flow. Procedimento realizado para o restabelecimento imediato da dimensão vertical de oclusão (DVO), diagnóstico funcional, e tratamento estético com excelente prognóstico. Além disto, este caso clínico apresenta e discute os aspectos gerais acerca da importância, indicação, aplicabilidade e condução clínica do mesmo. A REFRCP é um tratamento simplificado, seguro, reversível, conservador, de baixo custo em comparação a próteses de cerâmicas, que satisfaz as necessidades clínicas rapidamente com alta previsibilidade, reprodutibilidade, longevidade e tem ampla aplicação dentro dos diferentes tratamentos de reabilitação oral, entretanto, trata-se de uma técnica "operador-sensível" que requer certa habilidade e treino por parte do cirurgião dentista, um correto e minucioso enceramento diagnóstico e materiais de impressão com alta reprodução de detalhes e estabilidade dimensional.
\end{abstract}

Palavras-chave: Resinas compostas; Elastômeros de silicone; Dimensão vertical.

\section{Abstract}

The current development and reliability of adhesive systems and composite resins offer good options for treating and managing the loss of tooth structures. Patients with minimal, moderate or severe wear can be treated minimally invasively with adhesive composite restorations. Thus, according to the knowledge from clinical practice and based on the scientific literature on the topic discussed; and considering the different possibilities of conducting the case 
presented, it was proposed to carry out the rehabilitation of the maxilla and mandible with the use of Aesthetic and Functional Rehabilitation with Pressed Composite Resins (AFRPCR) with heated conventional viscosity composite resin and flow composite resin. Procedure performed for the immediate restoration of the vertical dimension of occlusion (VDO), functional diagnosis, and aesthetic treatment with excellent prognosis. In addition, this clinical case presents and discusses the general aspects about its importance, indication, applicability and clinical management. AFRPCR is a simplified, safe, reversible, conservative, low-cost treatment compared to ceramic prostheses, which meets clinical needs quickly with high predictability, reproducibility, longevity and has wide application within different oral rehabilitation treatments, however, it is an "operator-sensitive" technique that requires a certain skill and training on the part of the dental surgeon, a correct and meticulous diagnostic wax-up and impression materials with high reproduction of details and dimensional stability.

Keywords: Composite resins; Silicone elastomers; Vertical dimension.

\section{Resumen}

El actual desarrollo y confiabilidad de los sistemas adhesivos y de las resinas compuestas ofrecen buenas opciones para el tratamiento y manejo de las pérdidas de estructuras dentarias. Pacientes con mínimo, moderado o severo desgaste pueden ser tratados de forma mínimamente invasiva con restauraciones adhesivas en resina compuesta. Así, de acuerdo con los conocimientos a partir de la práctica clínica y con base en la literatura científica sobre el tema abordado, y considerando las diferentes posibilidades de realización del caso presentado, se propone una rehabilitación de la maxila y la mandíbula con el uso de la Rehabilitación Estética y Funcional con Resinas Compuestas Prensadas (REFRCP) con resina compuesta de viscosidad convencional calentada y resina compuesta flow. Procedimiento realizado para el restablecimiento inmediato de la dimensión vertical de la oclusión (DVO), diagnostico funcional y tratamiento estético con excelente pronostico. Además, este caso clínico presenta y discute los aspectos generales acerca de la importancia, indicación, aplicación y procedimiento clínico del mismo. La REFRCP es un tratamiento simplificado, seguro, reversible, conservador, de bajo costo en comparación a las prótesis cerámicas, que satisface las necesidades clínicas rápidamente con alta previsibilidad, reproductibilidad, longevidad y tiene amplia aplicación dentro de los diferentes tratamientos de rehabilitación oral, sin embargo, se trata de una técnica "operador-sensible" que requiere de cierta habilidad y entrenamiento por parte del odontólogo, un correcto y minucioso encerado de diagnostico y materiales de impresión con alta reproducción de detalles y estabilidad dimensional.

Palabras clave: Resinas compuestas; Elastómeros de silicona; Dimensión vertical.

\section{Introdução}

Atualmente, na oposição dos eficientes programas preventivos de saúde bucal, onde de modo geral a incidência das doenças orais infecciosas torna-se cada vez mais baixa na população mundial, os acometimentos bucais não infecciosos, como os distúrbios oclusais, são cada vez mais frequentes. O desgaste dentário é a perda progressiva da dureza dos tecidos dentários através de quatro processos (Shellis \& Addy, 2014). Os três primeiros são processos mecânicos: abrasão (desgaste produzido pela interação entre os dentes e outros materiais), abfração (trauma oclusal) e atrito (desgaste pelo contato dente-dente). Estes podem contribuir simultaneamente para o desgaste oclusal ou cervical (Kaidonis, 2008). O quarto processo, a erosão, é causada principalmente pela desmineralização do tecido duro por substâncias ácidas (Shellis \& Addy, 2014).

A perda da superfície dentária é um processo fisiológico normal que ocorre ao longo da vida (Poyser et al., 2005). No entanto, se a taxa de desgaste desafiar a viabilidade dos dentes, então ela é considerada patológica (Kelleher \& Bishop, 1999; Davies, Gray \& Qualtrough, 2002). O desgaste oclusal leva a uma redução no comprimento do dente e mudanças dimensionais significativas na morfologia facial (Davies, Gray \& Qualtrough, 2002). A perda da vertical causa um fechamento excessivo, que empurra a mandíbula para cima com força para manter o contato com os dentes superiores (Nanda, Jain \& Srivastava, 2011). Isso leva ao fechamento gradual do espaço entre a cabeça do côndilo e o disco articular causando alterações degenerativas, acompanhadas de dor e desconforto durante os movimentos mandibulares (Nanda, Jain \& Srivastava, 2011). Se a dimensão vertical de oclusão (DVO) não for restabelecida, a condição pode piorar. Assim, a reabilitação oral (através da reconstrução dental e do consequente restabelecimento da DVO) torna-se cada vez mais necessária para a recuperação da função e da estética do terço inferior da face.

Com os avanços nas ciências dos materiais, os compósitos evoluíram para demonstrar maior resistência ao desgaste, 
estabilidade de longo prazo intraoral e melhor adesão aos substratos dentais quando utilizados como restaurações diretas à substituição do amálgama dental (Gegauff, 1995; Fahl Júnior, 1996; Nixon, 1997). Entre suas muitas características favoráveis, os compósitos têm se mostrado um material ideal para longo prazo (Gegauff, 1995; Nixon, 1997). Para o uso da resina composta em uma reabilitação oral o grande obstáculo é a habilidade manual de conseguir reconstruir exatamente na boca do paciente em resina composta o que foi planejado no enceramento diagnóstico. Uma ferramenta que ajuda a transferir esse formato do enceramento para a boca do paciente é a matriz transparente de silicona por adição. Contudo a viscosidade da resina composta torna difícil a remoção dos excessos, levando a tempo clínico extenso e podendo gerar alterações dimensionais por dificuldade do escoamento do excesso do compósito. Por esse motivo que na técnica Bonded Funcional Esthetic Prototype (BFEP) a escolha foi por uma resina composta flow (McLaren, 2013). A baixa viscosidade da resina composta flow facilita a confecção da restauração, entretanto possui propriedades mecânicas inferiores quando comparado a resina composta de viscosidade convencional, assim torna-se um tratamento temporário, o qual é realizado anteriormente a reabilitação com restaurações cerâmicas. O presente caso clínico propôs-se a realização da reabilitação da maxila e de mandíbula com o uso da Reabilitação Estética e Funcional com Resinas Compostas Prensadas (REFRCP) com resina composta de viscosidade convencional aquecida e resina composta flow.

\section{Metodologia}

O presente artigo trata-se de um relato de caso clínico (estudo de caso) com fins descritivos, exploratórios, com abordagem qualitativa, de acordo com estudo anterior (Pereira et al., 2018; Costa et al., 2020). Após realização do plano de tratamento, o paciente M.V.J. assinou o Termo de Consentimento Live e Esclarecido (TCLE), conforme estudo prévio (Almeida et al., 2020). Assim, segundo os conhecimentos, prática clínica e com base na literatura científica sobre o tema abordado, e considerando as diferentes possibilidades de condução do caso apresentado, propôs-se a realização da reabilitação da maxila e de mandíbula com o uso da Reabilitação Estética e Funcional com Resinas Compostas Prensadas (REFRCP) com resina composta de viscosidade convencional aquecida e resina composta flow. Procedimento realizado para o restabelecimento imediato da DVO, diagnóstico funcional, e tratamento estético com excelente prognóstico. Além disto, este caso clínico apresenta e discute os aspectos gerais acerca da importância, indicação, aplicabilidade e condução clínica do mesmo.

\section{Relato de Caso}

Paciente M.V.J., 37 anos, sexo masculino, procurou atendimento odontológico com queixa estética, desgastes e sensibilidade dental. O mesmo relatou ter realizado tratamento ortodôntico na adolescência devido a agenesia dos incisivos laterais superiores (Figura 1), ausência de sintomatologia nas articulações temporomandibulares ou dores tencionais.

Figura 1 - Vista lateral direita (A) e esquerda (B).
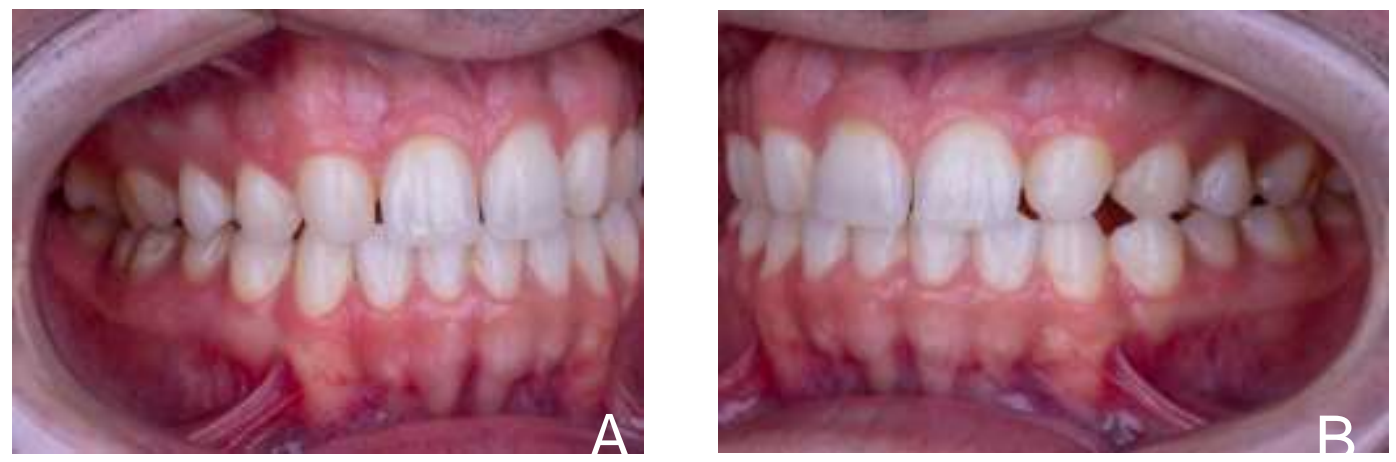

Fonte: Autores. 
Ao exame clínico (Figura 1), foi verificado que a ortodontia tracionou os caninos para a região dos incisivos laterais deixando alguns diastemas, sendo assim, com perda das guias de lateralidade em caninos, consequentemente levando as em grupo em ambos os lados gerando forças horizontais indesejáveis na região posterior, causando abfrações (lesões cervicais não cariosas por trauma oclusal) em alguns dentes devido a oclusão desequilibrada.

Foi utilizado dispositivo interoclusal rígido (placa acrílica) durante 03 meses para controle da parafunção, devolvendo o tônus muscular para restituir a DVO perdida devido ao desgaste dental. As moldagens foram realizadas com silicona reação por adição (Variotime Easy Putty; Kulzer GmbH, Hanau, Alemanha) nas consistências denso / leve pela técnica da dupla impressão, juntamente com o registro interoclusal com DVO restabelecida, e a tomada do arco facial que foram montados em articulador semi-ajustável, assim foi confeccionado enceramento de diagnóstico 0,5 $\mathrm{mm}$ a 1,0 mm aquém da margem gengival (Figura 2). Os segundos moldares não foram encerados para servirem de apoio e orientação para a estabilização da matriz transparente, sendo que após o término do restabelecimento da DVO, também foram incluídos na reabilitação.

Figura 2 - Modelos encerados montados em articulador semi-ajustável.

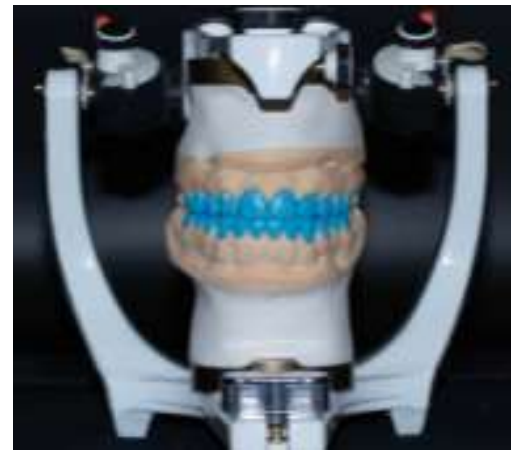

Fonte: Autores.

Foi realizado a prova do tratamento proposto antes de executar o tratamento final através do ensaio do enceramento, também chamado de Mock Up, apara avaliação estética e funcional diretamente na boca do paciente (Figura 3).

Figura 3 - Vista frontal dos dentes em oclusão (A) e maxilares abertos (B).
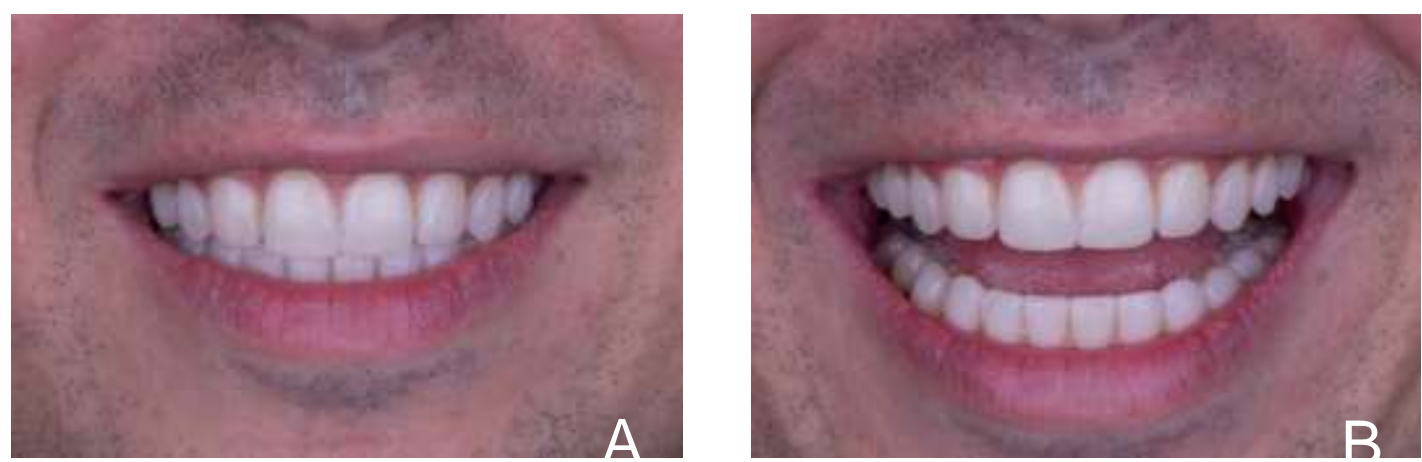

Fonte: Autores.

Após a aprovação do ensaio, foi confecionada uma matriz transparente com silicona de adição (Elite Glass; Zhermack, Badia Polesine, RO, Italy), para servir de molde para inserção da resina composta. A resina composta Z350 (3MESPE, St. Paul, MN, EUA) na cor A1E foi aquecida em aparato (Calset Warmer for Composites; Addent, Danbury, CT, EUA) por 3 min à temperatura de $54^{\circ} \mathrm{C}$ e inserida na matriz transparente envolvendo quase todo o molde das faces do dente promovido pelo enceramento do modelo, mas deixando um pequeno espaço entre o esmalte do dente a ser restaurado e a resina 
composta Z350. Este pequeno espaço foi preenchido na matriz transparente pela inserção de um fino incremento da resina composta Tetric N Flow (Ivoclar Vivadent, Schaan, Liechtenstein) na cor Bleach L sem prévio aquecimento. A sequência dental para tratamento foi inserção da matriz transparente carregada com as resinas compostas em um dos incisivos centrais, depois o outro incisivo central e posteriormente segue realizando a inserção em dentes análogos de dois em dois, na sequência: incisivos laterais, caninos, primeiros pré-molares segundos pré-molares e primeiros molares. Sempre isolando os dentes vizinhos para facilitar o acabamento proximal.

A sequência da técnica restauradora foi: 1- Isolamento relativo com um afastador de lábios e bochecha OptraGate (Ivoclar Vivadent); 2- Alívio de ponto de contato, se necessário, com fina lixa diamantada (Diamond Strip; TDV, Pomerode, SC, Brasil); 3- Profilaxia com taça de borracha (Polidor Jiffy Polisher Cup; Ultradent, South Jordan, UT, EUA) montada em contra ângulo com pasta com Clorexidina 2\% (Concepsis Scrub; Ultradent); 4- Isolamento dos dentes vizinhos com envolvimento das papilas interdentais com fita de teflon (Tigre, Castro, PR, Brasil); 5- Condicionamento ácido do esmalte por $30 \mathrm{~s}$ e da dentina por $15 \mathrm{~s}$ das faces que serão restauradas (Ultra Etch; Ultradent); 6- Lavar por no mínimo 30 s e depois secar de maneira seletiva, a dentina deve permanecer úmida; 7- Aplicação do primer (Optibond FL Prime; Kerr, Romulus, MI, EUA), e leve jato de ar por 5 s; 8- Aplicação do adesivo Optibond FL (Kerr, Romulus, MI, EUA) seguido de polimerização por $10 \mathrm{~s}$ com fotoativador (Valo; Ultradent); 9- Inserção da matriz transparente carregada com as resinas compostas posicionada no local correto com leve pressão seguido de polimerização por $40 \mathrm{~s}$ com fotoativador (Valo; Ultradent); 10- O refino do ajuste final da oclusão foi executado com pontas diamantadas de fina e ultra granulação (Jota AG, Rüthi, Suíça) em relação cêntrica, de forma criteriosa, sendo também verificados os movimentos de protrusão e lateralidade; 11 - Acabamento com lamina de bisturi 12 (Swann-Morton, Sheffield, Reino Unido), pontas diamantadas de fina e ultra granulação (Jota AG) e lixa de acabamento proximal para resina (Sof-Lex; 3M-ESPE); 12- Polimento 24 horas após fotoativação das resinas compostas com polidor espiral (9150.RA.140 / 9837.RA.140; Jota).

$\mathrm{O}$ aspecto final está evidenciado nas Figuras 4 e 5.

Figura 4 - Vista lateral direita (A) e esquerda (B) após ao término do tratamento.
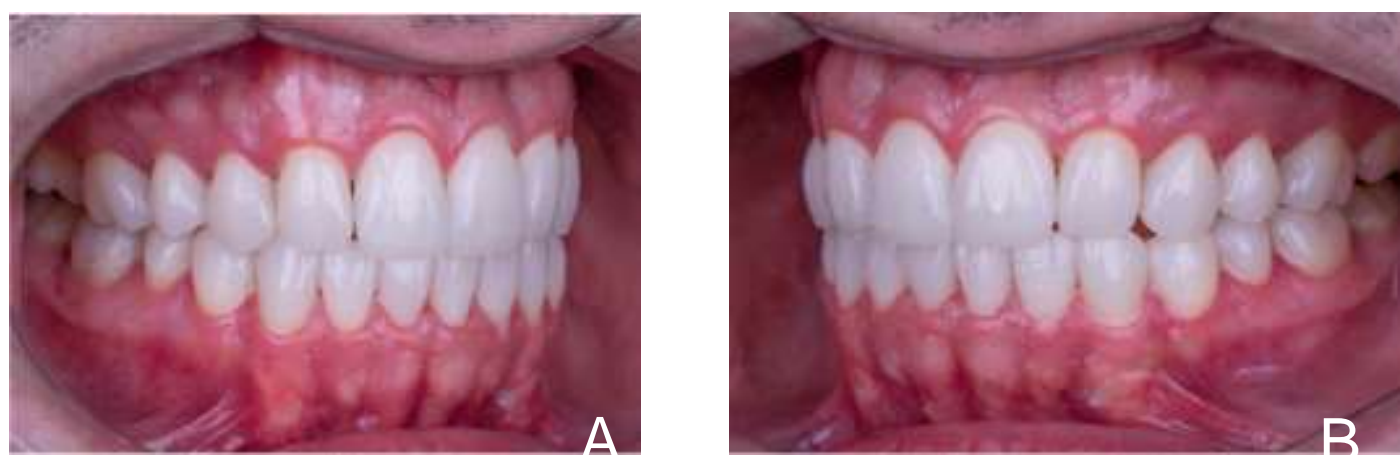

Fonte: Autores. 
Figura 5 - Vista frontal antes da realização do tratamento (A) e depois do tratamento evidenciando-se a forma, textura, cor e harmonia dos dentes.
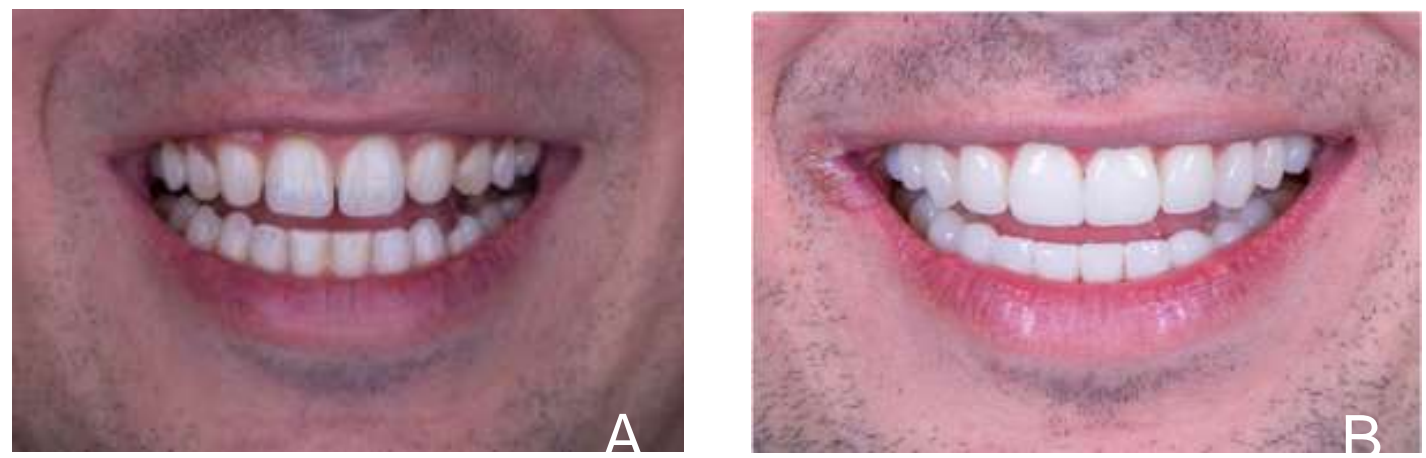

Fonte: Autores.

\section{Discussão}

Alguns estudos recomendam o pré-aquecimento de materiais à base de resina antes de sua aplicação (Daronch, Rueggeberg \& Goes, 2005; Daronch, Rueggeberg \& Goes, 2006; Mundim et al., 2011). O a energia gerada pelo préaquecimento do material aumenta a taxa de colisão entre grupos não reativos e radicais, o que resultaria em uma reação de polimerização mais completa (Daronch, Rueggeberg \& Goes, 2005; Daronch, Rueggeberg \& Goes, 2006; Mundim et al., 2011). Os benefícios dos compostos de pré-aquecimento podem ter um impacto sobre procedimentos restauradores, com a aplicação de menor exposição à luz para fornecer valores de conversão semelhantes a aqueles vistos em condições não aquecidas (Daronch, Rueggeberg \& Goes, 2005).

Além disto, estudos anteriores sugeriram que o manuseio destes materiais resinosos a temperaturas elevadas, variando de $50^{\circ} \mathrm{C}$ a $60^{\circ} \mathrm{C}$ poderia resultar em melhores taxas de conversão, independentemente das condições de polimerização (Teixeira et al., 2021). O presente caso clínico utilizou $54^{\circ} \mathrm{C}$ obtendo excelente estética (Figura 5B). O pré-aquecimento de compósitos antes da fotoativação geralmente diminui sua viscosidade, melhorando a adaptação marginal e reduzindo a microinfiltração (Teixeira et al., 2021). Além disso, o aumento da temperatura de polimerização aumenta mobilidade radical e monomérica, resultando em maior conversão, que por sua vez pode promover propriedades físicas e mecânicas aprimoradas de compósitos pré-aquecidos, como maior dureza superficial e maior resistência à flexão e resistência à tração (Calheiros et al., 2014; Lima et al., 2018: Chaharom et al., 2020). Assim, o presente estudo com a técnica da REFRCP, apresentou forma, textura e cor harmônicos promovendo um sorriso com resultado estético excelente, quando comparado ao quadro inicial, que satisfez o paciente e o cirurgião dentista. Entretanto, por se tratar se tratar de um tratamento final, novo relato de caso poderia ser realizado após alguns anos do término de tratamento em estudos futuros para validação da técnica.

\section{Considerações Finais}

A REFRCP é um tratamento simplificado, seguro, reversível, conservador, de baixo custo em comparação a próteses de cerâmicas, que satisfaz as necessidades clínicas rapidamente com alta previsibilidade, reprodutibilidade, longevidade e tem ampla aplicação dentro dos diferentes tratamentos de reabilitação oral, entretanto, trata-se de uma técnica "operador-sensível" que requer certa habilidade e treino por parte do cirurgião dentista, um correto e minucioso enceramento diagnóstico e materiais de impressão com alta reprodução de detalhes e estabilidade dimensional. 


\section{Agradecimentos}

O presente trabalho foi realizado com apoio da Coordenação de Aperfeiçoamento de Pessoal de Nível Superior Brasil (CAPES) - Código de Financiamento 001.

\section{Referências}

Almeida, R. R., Carvalho, G. A. O., Câmara, J. V. F., \& Pierote, J. J. A. (2020). Composite resin restoration for diastema closure: case report. Research, Society and Development, 9(7), e644974703.

Calheiros, F. C., Daronch, M., Rueggeberg, F. A., \& Braga, R. R. (2014). Effect of temperature on composite polymerization stress and degree of conversion. Dental Materials, 30(6), 613-618.

Chaharom, M. E. E., Bahari, M., Safyari, L., Safarvand, H., Shafaei, H., Jafari Navimipour, E., Alizadeh Oskoee, P., Ajami, A. A., \& Abed Kahnamouei, M. (2020). Effect of preheating on the cytotoxicity of bulk-fill composite resins. Journal of Dental Research Dental Clinics Dental Prospects, 14(1), 19-25.

Costa, F. A. d. N., Cruz, J. H. d. A., Sá, E. T. F. d., Palmeira, J. T., Pontes, N. G., Oliveira, B. F. d., Silva, R. L. B. d., Pereira, V. A. C., Rolim, A. K. A., \& Figueirêdo Júnior, E. C. (2020). Aesthetic restoration with fiberglass pin and compound resin: clinical case report. Research, Society and Development, 9(7), e810974899.

Daronch, M., Rueggeberg, F. A., \& De Goes, M. F. (2005). Monomer conversion of pre-heated composite. Journal of Dental Research, 84(7), 663-667.

Daronch, M., Rueggeberg, F. A., De Goes, M. F., \& Giudici, R. (2006). Polymerization kinetics of pre-heated composite. Journal of Dental Research, 85(1), $38-43$.

Davies, S. J., Gray, R. J., \& Qualtrough, A. J. (2002). Management of tooth surface loss. British Dental Journal, 192(1), 11-16, 19-23.

Fahl Júnior, N. (1996). The direct/indirect composite resin veneers: a case report. Practical Periodontics and Aesthetic Dentistry, 8(7), 627-638; quiz 640.

Gegauff, A. G. (1995). Provisional restorations. In Rosensteil SF, Land MF, Fujimoto J, eds. Contemporary Fixed Prosthodontics. (2 ed., pp. 325,329). MO: Mosby-Year Book.

Kaidonis, J. A. (2008). Tooth wear: the view of the anthropologist. Clinical Oral Investigations, 12 Suppl 1(Suppl 1), S21-26.

Kelleher, M., \& Bishop, K. (1999). Tooth surface loss: an overview. British Dental Journal, 186(2), 61-66.

Lima, M. O., Catelan, A., Marchi, G. M., Lima, D. A., Martins, L. R., \& Aguiar, F. H. (2018). Influence of pre-heating and ceramic thickness on physical properties of luting agents. Journal of Applied Biomaterials \& Functional Materials, 16(4), 252-259.

McLaren, E. A. (2013). Bonded functional esthetic prototype: an alternative pre-treatment mock-up technique and cost-effective medium-term esthetic solution. The Compendium of Continuing Education in Dentistry, 34(8), 596-607.

Mundim, F. M., Garcia Lda, F., Cruvinel, D. R., Lima, F. A., Bachmann, L., \& Pires-de-Souza Fde, C. (2011). Color stability, opacity and degree of conversion of pre-heated composites. Journal of Dentistry, 39 Suppl 1, e25-29.

Nanda, A., Jain, V., \& Srivastava, A. (2011). An electromyographic study to assess the minimal time duration for using the splint to raise the vertical dimension in patients with generalized attrition of teeth. Indian Journal of Dental Research Indian, 22(2), $303-308$.

Nixon, R. L. (1997). Provisionalization for ceramic laminate veneer restorations: a clinical update. Practical Periodontics and Aesthetic Dentistry, 9(1), 17-27; quiz 28.

Pereira, A. S., Shitsuka, D. M., Parreira, F. J., \& Shitsuka, R. (2018). Metodologia da pesquisa científica. UFSM.

Poyser, N. J., Porter, R. W., Briggs, P. F., Chana, H. S., \& Kelleher, M. G. (2005). The Dahl Concept: past, present and future. British Dental Journal, 198(11), 669-676; quiz 720.

Shellis, R. P., \& Addy, M. (2014). The interactions between attrition, abrasion and erosion in tooth wear. Monographs in Oral Science, 25, 32-45.

Teixeira, J. P. S., Cavalcante, S. K. d. S., Roncolato, Á. T. L., Mendes, T. A. D., Pinto, A. C. M. D., Brito, R. d. S., Fontes, N. M., Silva, R. A. D. A. d., Isaias, P. H. C., \& Dinelly, É. M. P. (2021). Use of preheated resin as a cementitious material in indirect restoration: A literature review. Research, Society and Development, 10(7), e2810716293. 